\title{
COMPOSIÇÃO QUÍMICA DA CASCA DE OVO DE GALINHA EM PÓ
}

\author{
JULIANA DOS SANTOS VILAR* \\ ARMANDO UBIRAJARA OLIVEIRA SABAA-SRUR** \\ RUY GARCIA MARQUES***
}

\begin{abstract}
A casca de ovo de galinha constitui subproduto utilizado no preparo de multimisturas alimentares distribuídas no Brasil. A carência de informações químicas sobre a casca de ovo motivou a realização deste trabalho que visou desenvolver tecnologia para obtenção desse produto na forma de pó e determinar o seu conteúdo de nutrientes com vistas a sua utilização como fonte de cálcio. Depois de higienizadas e desidratadas, as cascas foram transformadas em pó com auxílio de moinho, tendo sido obtido rendimento de 98,45 $\pm 0,70 \%$. Determinações analíticas revelaram que $100 \mathrm{~g}$ desse produto continha baixo teor de umidade $(1,3490 \pm 0,0274 \mathrm{~g})$, resíduo mineral fixo equivalente a $91,9600 \pm 0,2218 \mathrm{~g}$ e baixo teor calórico $(30,6823$ \pm 4,9069 Kcal), provenientes de 4,3693 $\pm 0,3977 \mathrm{~g}$ de proteínas, 0,7837 $\pm 0,0560 \mathrm{~g}$ de lipídios e 1,5379 $\pm 0,7029 \mathrm{~g}$ de carboidratos totais, além de $30.263,72 \mathrm{mg}$ de cálcio. Considerando que a ingestão diária de cálcio recomendada para adultos saudáveis é de $1000 \mathrm{mg}$, apenas $3,30 \mathrm{~g}$ de casca de ovo em pó supririam essa necessidade. Os resultados obtidos sugerem que esse subproduto constitui potencial fonte de cálcio para a alimentação, sendo necessários maiores estudos sobre a sua biodisponibilidade em humanos.
\end{abstract}

PALAVRAS-CHAVE: CASCA DE OVO; CÁLCIO; Gallus gallus.

* $\quad$ Mestre em Ciência de Alimentos, Universidade Federal Rural do Rio de Janeiro, Rio de Janeiro, RJ (email: dravilar@yahoo.com.br).

** Doutor em Ciência de Alimentos, Professor Assistente, Departamento de Nutrição Básica e Experimental, Instituto de Nutrição Josué de Castro, Universidade Federal do Rio de Janeiro, Rio de Janeiro, RJ (e-mail: sabaasrur@yahoo.com.br).

*** Doutor em Técnica Cirúrgica e Cirurgia Experimental, Professor Adjunto, Departamento de Cirurgia Geral, Faculdade de Ciências Médica, Universidade Estadual do Rio de Janeiro, Rio de Janeiro, RJ (e-mail: ruymarques@globo.com). 


\section{INTRODUÇÃO}

Apesar de apresentarem teores consideráveis de vitaminas e sais minerais, partes não tradicionais de matérias-primas são geralmente desprezadas na alimentação. Essas porções são incluídas na alimentação denominada alternativa por apresentarem potencial benéfico à nutrição humana. Além disso, assumem importância econômica, social e ambiental, devido ao aproveitamento de subprodutos que comumente são utilizados como ração animal ou descartados como resíduo industrial.

A casca de ovo de galinha é totalmente desprezada durante as preparações culinárias pelas Unidades de Alimentação Coletivas no decorrer da elaboração das refeições e pelas indústrias nos processos fabris para produção de maioneses, ovos pasteurizados ou em pó.

A média per capta de consumo de ovo integral no Brasil foi de 141 unidades em 2006 (UBA, 2006) e sua produção atingiu cerca de 20.470 bilhões de unidades no ano de 2008 (IBGE, 2008). Isso representou 102,35 toneladas de casca de ovos, que provavelmente foi desprezada no ambiente.

A casca de ovo constitui um dos ingredientes de multimisturas alimentares distribuídas no Brasil por organizações não governamentais como estratégia de combate à fome e à desnutrição. Apesar da carência de dados sobre a sua qualidade nutricional, sabe-se que o cálcio encontra-se presente na forma de carbonato de cálcio na proporção de 40\% do produto (NAVES, 2003).

O cálcio assume extrema importância para o organismo, sobretudo para a mineralização óssea. A única fonte disponível desse mineral para o organismo humano provém da alimentação mediante consumo de produtos naturalmente ricos em cálcio, tais como o leite e seus derivados. Também se pode recorrer aos alimentos fortificados com cálcio, sendo importante prover adequada ingestão do mineral para o completo crescimento e maturação óssea (WEAVER e HEANEY, 2003). A necessidade diária desse mineral ainda pode ser suprida pela ingestão de suplementos farmacológicos. Os suplementos de cálcio são geralmente apresentados na forma de sais ou combinações de sais, como carbonato, citrato, lactato e fosfato, e em menor proporção gluconato, glubionato, gluceptato. O conteúdo de cálcio de tais suplementos varia, havendo maior percentual de carbonato de cálcio $\left(\mathrm{CaCO}_{3}\right)$ que contém $40 \%$ de cálcio. Outros sais, como citrato, lactato e gluconato fornecem 21\%, 14\% e 9,3\% de cálcio, respectivamente (FISHBEIN, 2004).

A casca de ovo, subproduto da industrialização dessa matéria-prima contém elevado teor de cálcio e poderia representar mais uma opção para atender as necessidades nutricionais da população. Dessa forma, o presente trabalho objetivou o desenvolvimento de tecnologia de obtenção de cascas de ovos de galinha em pó, além de determinar a sua composição centesimal e seu perfil de minerais e aminoácidos essenciais.

\section{MATERIAL E MÉTODOS}

Cascas de ovos de galinha (Gallus gallus, L.), oriundas de processo fabril, foram fornecidas pela indústria de alimentos Sohovos AB Brasil - Sorocaba (São Paulo). As amostras foram coletadas, acondicionadas em caixas térmicas de Isopor ${ }^{\circledR}$ com gelo seco e transportadas até o Laboratório de Processamento de Alimentos do Instituto de Nutrição Josué de Castro da Universidade Federal do Rio de Janeiro.

Após a chegada ao laboratório, as cascas de ovos foram higienizadas com solução clorada e submetidas à secagem em estufa com circulação de ar aquecido a $55 \pm 5^{\circ} \mathrm{C}$. Depois de desidratadas, foram trituradas com auxílio de moinho de facas até que as partículas atravessassem peneiras de malhas $0,25 \mathrm{~mm}$. O pó obtido foi acondicionado em sacos de polietileno com alta barreira ao vapor d'água e armazenado à temperatura ambiente na presença de luz.

O rendimento do processo de obtenção da casca de ovo em pó foi calculado pela razão entre a massa de cascas de ovos em pó produzida e a massa de cascas de ovos antes da secagem (ORNELLAS, 2001). 
Para a determinação da composição centesimal do produto obtido foram mensurados os teores de umidade, cinzas, extrato etéreo total, proteína bruta e fração Nifext, conforme metodologias preconizadas nas Normas Analíticas do Instituto Adolfo Lutz (IAL, 2005), sendo os resultados expressos em g/100 g de casca de ovo em pó. O valor energético total foi calculado pela soma dos produtos do número de gramas de proteínas e de carboidratos multiplicado por quatro (4 kcal/g), e do número de gramas de lipídios multiplicado por nove $(9 \mathrm{kcal} / \mathrm{g})$, sendo seu resultado expresso em quilocalorias (kcal/100 g) (KRAUSE e MAHAN, 2002).

Para a determinação do perfil de minerais, as amostras da casca de ovo em pó foram calcinadas em mufla a $550^{\circ} \mathrm{C}$ e as cinzas obtidas dissolvidas em $\mathrm{HCl} 2 \mathrm{~mol} / \mathrm{L}$, sendo analisadas por espectrometria de massa com plasma indutivamente acoplado no modo semi-quantitativo, utilizando-se o equipamento ELAN 6000 da Perkin Elmer-Sciex (AOAC, 2000). Os resultados foram expressos em mg do mineral correspondente/100 $\mathrm{g}$ de casca de ovo em pó.

Determinou-se o perfil de aminoácidos essenciais segundo metodologia preconizada pela Association of Official Analytical Chemists (AOAC, 2000). Os aminoácidos foram obtidos após hidrólise de alíquotas de casca de ovo em pó com $\mathrm{HCl} 6 \mathrm{M}$ por $24 \mathrm{~h}$ a $110 \pm 1^{\circ} \mathrm{C}$ em ampolas seladas a vácuo. Os hidrolisados foram evaporados em dessecador contendo pastilhas de $\mathrm{NaOH}$ e posteriormente suspensos em tampão citrato $\mathrm{pH}$ 2,2. Realizou-se a determinação por cromatografia a líquido de alta eficiência com detecção fluorimétrica, após derivatização com 6-aminoquinolyl-N-succinimidyl carbamate. Usouse coluna de resina de troca catiônica e derivatização pós-coluna com ninidrina em auto-analisador de aminoácidos Hewlett Packard, modelo 1090M, equipado com coluna de $200 \mathrm{~mm}$ de comprimento, contendo resina de troca iônica de sódio, com injeção de $25 \mu \mathrm{L}$ da amostra e operando em condições para hidrolisados protéicos (fluxo de $1 \mathrm{~mL} / \mathrm{min}$, à temperatura de $25^{\circ} \mathrm{C}$ ) (SPACKMAN, STEIN e MOORE, 1958). Os resultados foram expressos em $\mathrm{g}$ de aminoácidos por $100 \mathrm{gramas}$ (g/100 g).

Os resultados obtidos foram analisados por meio de estatísticas descritivas, usando-se o software Microsoft Excel 2007 (TRIOLA, 2005).

\section{RESULTADOS E DISCUSSÃO}

O processo de obtenção da casca de ovo em pó apresentou elevado rendimento (98,45\%), cuja pequena perda do material significa menor volume de resíduo descartado no ambiente, caso esse produto venha a ser incorporado à alimentação humana.

Os resultados de composição centesimal e o valor energético total (VET) da casca de ovo em pó encontrados no presente estudo e no trabalho de Sant'Ana et al. (2000) estão apresentados na Tabela 1.

\section{TABELA 1 - COMPARAÇÃO DOS RESULTADOS DE COMPOSIÇÃO CENTESIMAL E VET ENCONTRADOS NA CASCA DE OVO DE GALINHA EM PÓ COM OS RESULTADOS OBTIDOS POR SANT'ANA et al. (2000)}

\begin{tabular}{c|c|c}
\hline Parâmetros analisados & $\begin{array}{c}\text { Resultados obtidos } \\
(\mathrm{X} \pm \mathrm{DP})\end{array}$ & $\begin{array}{c}\text { Resultados encontrados } \\
\text { por Sant'Ana et al., 2000 } \\
(\mathrm{X} \pm \mathrm{DP})\end{array}$ \\
\hline Umidade $\left(\mathrm{g} .100 \mathrm{~g} \mathrm{~g}^{-1}\right)$ & $1,3490 \pm 0,0274$ & $0,63 \pm 0,01$ \\
Cinzas $\left(\mathrm{g} .100 \mathrm{~g}^{-1}\right)$ & $91,9600 \pm 0,2218$ & $87,71 \pm 1,75$ \\
Proteínas $\left(\mathrm{g} .100 \mathrm{~g}^{-1}\right)$ & $4,3693 \pm 0,3977$ & $4,00 \pm 0,16$ \\
Carboidratos totais $\left(\mathrm{g} .100 \mathrm{~g}^{-1}\right)$ & $1,5379 \pm 0,7029$ & 8,11 \\
Lipídios totais $\left(\mathrm{g} .100 \mathrm{~g}^{-1}\right)$ & $0,7837 \pm 0,0560$ & $0,09 \pm 0,01$ \\
Valor energético total & $30,6823 \pm 4,9069$ & 49,25 \\
(Kcal.100 g $\left.\mathrm{g}^{-1}\right)$ &
\end{tabular}

$\mathrm{X}=$ média e DP = desvio-padrão. 
Os resultados mostraram que o produto contém baixo teor de umidade que contribui para impedir a proliferação de microorganismos, além de facilitar seu uso em formulações desidratadas como, por exemplo, as preparações para obtenção de sopas, pós para pudins, bebidas lácteas, encapsulados, entre outros produtos.

Deve-se ressaltar que a maior fração encontrada na casca de ovo em pó foi a de cinzas e, dessa forma, o produto pode ser considerado uma das maiores fontes naturais de minerais. No entanto, estudos devem ser realizados para avaliar a biodisponibilidade desses minerais para os humanos. O conteúdo de lipídios encontrado nesse produto pode ser considerado irrelevante e o reduzido valor energético total favorece dietas hipocalóricas que necessitem de complementação ou suplementação de minerais.

A fração Nifext da casca de ovo não deveria ser considerada como carboidrato por tratar-se de produto de origem animal, que tem como característica baixo teor ou ausência desse composto. Sant'Ana et al. (2000) consideraram a fração Nifext como carboidratos e obtiveram elevado teor de carboidratos totais em comparação aos resultados do presente estudo.

Os resultados da composição centesimal obtidos nesta pesquisa mostraram-se semelhantes aos encontrados por Sant'Ana et al. (2000) no que diz respeito aos teores de umidade, cinzas, proteínas e lipídios, diferindo quanto ao VET em razão dos valores de carboidratos totais. Também deve ser considerado que esses valores podem ser alterados devido a fatores que influenciam a composição do ovo, tais como seu tamanho, alimentação e estado sanitário das aves.

Apesar da casca de ovo em pó apresentar certo teor de proteína bruta, pesquisas precisam ser realizadas sobre seu perfil de aminoácidos, assim como para determinar seu coeficente de eficácia proteica. Tais parâmetros são importantes para definir a qualidade biológica e uso dessa fração, já que se trata de resíduo de natureza animal e as porções proteicas procedentes dessa origem tendem a revelar proteínas de alto valor biológico.

O uso de alimento como fonte proteica depende de fatores como a concentração total de proteínas e a proporção de aminoácidos essenciais presente nas proteínas em questão (ANDRIGUETTO et al., 1999). Para avaliar a qualidade química das proteínas presentes na casca de ovo foi determinado seu perfil de aminoácidos essenciais, cujos resultados encontram-se na Tabela 2.

TABELA 2 - PERFIL DE AMINOÁCIDOS ESSENCIAIS PRESENTES NA CASCA DE OVO EM PÓ E COMPARAÇÃO COM A PROTEÍNA PADRÃO ESTABELECIDA PELA FAO (1985)

\begin{tabular}{ccc}
\hline $\begin{array}{c}\text { Aminoácidos (AA) } \\
\text { essenciais }\end{array}$ & g AA100 g Proteína Padrão & g de AA $\mathbf{1 0 0}$ g casca de ovo em pó \\
\hline Histidina & - & 0,43 \\
Isoleucina & 4 & 0,295 \\
Leucina & 7,00 & 0,44 \\
Lisina & 5,50 & 0,295 \\
Metionina & - & N.A. \\
Cisteína & - & N.A. \\
Sulfurados totais* & 3,50 & N.A. \\
Fenilalanina & - & 0,18 \\
Tirosina & - & 0,12 \\
Aromáticos totais & 6,00 & 0,3 \\
Treonina & 4,00 & 0,39 \\
Triptofano & 1,00 & N.A. \\
Valina & 5,00 & 0,61 \\
\hline
\end{tabular}

*aminoácidos sulfurados totais = metionina + cisteína.

**aminoácidos aromáticos totais = fenilalanina + tirosina.

N.A. = não analisado. 
Todos os aminoácidos avaliados mostraram concentrações inferiores quando comparados à proteína padrão sugerida pela FAO (1985). O produto não pode ser considerado como boa fonte de proteínas, mas deve ser estudado como possível fonte de cálcio para fenilcetonúricos devido ao baixo conteúdo de fenilalalina.

O tratamento da fenilcetonúria no que diz respeito à alimentação engloba a limitação da ingestão de proteínas de fontes naturais que apresentam alta concentração em fenilalanina. A restrição proteica natural deve ser compensada pela utilização de mistura de aminoácidos isenta ou com pequena quantidade de fenilalanina em sua composição. A mistura de aminoácidos é, portanto, essencial ao tratamento adequado da doença. Sua ausência impede o tratamento, pois ocasiona deficiência nutricional importante levando à quadros de desnutrição proteico-energética e de hipovitaminoses, entre outros, já que a dieta é extremamente restritiva sob vários aspectos (BRASIL, 2002).

O protocolo clínico e diretrizes terapêuticas para fenilcetonúria do Ministério da Saúde_estabelece que os produtos utilizados no seu tratamento são complementos alimentares e fórmulas de aminoácidos isentas de fenilalanina. A característica básica desses produtos é a concentração muito baixa de fenilalanina que não pode ser superior a $0,1 \mathrm{~g}$ ou $100 \mathrm{mg}$ de fenilalanina por $100 \mathrm{~g}$ do produto (BRASIL, 2002), valor próximo ao encontrado neste estudo.

Os resultados do perfil de minerais sugerem que a casca de ovo constitui fonte concentrada de minerais que deve ser nutricionalmente considerada, apesar da necessidade de estudos sobre a biodisponibilidade desses elementos. Dentre os minerais pesquisados, o cálcio apresentou o maior teor, verificando-se certa quantidade de magnésio, sódio, estrôncio, fósforo e potássio (Tabela 3).

TABELA 3 - VALORES MÉDIOS DO TEOR DE MINERAIS (mg/100 g) PRESENTE NA CASCA DE OVO

\begin{tabular}{|c|c|c|c|c|c|c|c|}
\hline Minerais & (mg/100g) & Minerais & (mg/100g) & Minerais & $(\mathrm{mg} / 100 \mathrm{~g})$ & Minerais & $(\mathrm{mg} / 100 \mathrm{~g})$ \\
\hline $\mathrm{Li}$ & 0,0061 & $\mathrm{Ga}$ & 0,0004 & $\mathrm{Sr}$ & 103,2 & CE & $<0,005$ \\
\hline $\mathrm{Be}$ & $<0,002$ & $\mathrm{Ge}$ & 0,0479 & Y & 0,001 & PR & $<0,0005$ \\
\hline $\mathrm{Na}$ & 117,475 & As & 0,012 & $\mathrm{Zr}$ & 0,0005 & $\mathrm{Nd}$ & $<0,001$ \\
\hline $\mathrm{Mg}$ & 351,1 & $\mathrm{Se}$ & 0,01 & $\mathrm{Nb}$ & $<0,001$ & SM & 0,002 \\
\hline $\mathrm{Al}$ & $<0,5$ & $\mathrm{Rb}$ & 0,040 & Mo & 0,023 & Eu & 0,0018 \\
\hline K & 41,971 & $\mathrm{~Tb}$ & $<0,0005$ & $\mathrm{Ru}$ & $<0,0001$ & $\mathrm{Gd}$ & $<0,0005$ \\
\hline $\mathrm{Ca}$ & 30263,72 & Dy & $<0,0005$ & $\mathrm{Pd}$ & 0,0684 & Ir & $<0,0003$ \\
\hline Sc & $<0,3$ & $\mathrm{Ho}$ & $<0,0005$ & $\mathrm{Ag}$ & $<0,002$ & PT & $<0,0004$ \\
\hline $\mathrm{Ti}$ & 0,458 & $\mathrm{Er}$ & $<0,0005$ & $\mathrm{Cd}$ & $<0,02$ & $\mathrm{Au}$ & 0,0001 \\
\hline V & 0,008 & $\mathrm{Tm}$ & $<0,0005$ & In & $<0,001$ & $\mathrm{Hg}$ & $<0,02$ \\
\hline $\mathrm{Cr}$ & $<0,1$ & $\mathrm{Yb}$ & $<0,0005$ & $U$ & $<0,001$ & $\mathrm{Tl}$ & 0,0014 \\
\hline $\mathrm{Mn}$ & 0,031 & Lu & $<0,0005$ & Sn & $<0,04$ & PB & $<0,02$ \\
\hline Fé & 1,5 & $\mathrm{Hf}$ & $<0,0005$ & $\mathrm{Te}$ & 0,0008 & $\mathrm{Bi}$ & $<0,02$ \\
\hline Co & 0,052 & $\mathrm{Ta}$ & $<0,0005$ & 1 & $<0,8$ & Th & $<0,0005$ \\
\hline $\mathrm{Ni}$ & $<0,0003$ & W & $<0,002$ & Cs & $<0,0005$ & $P$ & 98,2 \\
\hline $\mathrm{Cu}$ & $<0,0001$ & $\mathrm{Re}$ & $<0,0002$ & $\mathrm{Ba}$ & 3,9739 & $\mathrm{Si}$ & $<0,0004$ \\
\hline $\mathrm{Zn}$ & 0,083 & Os & $<0,0001$ & La & $<0,001$ & $\mathrm{Sb}$ & $<0,001$ \\
\hline
\end{tabular}

Em estudo realizado com multimistura, Sant'Ana et al. (2000) citaram que a casca de ovo apresenta composição similar, em termos de cálcio, ao resultado encontrado nesta pesquisa (30810 mg.100 g-1 e 30263,72 mg.100 g ${ }^{-1}$, respectivamente). A ingestão diária de cálcio recomendada para adultos é de $1000 \mathrm{mg}$ e para indivíduos com mais de 50 anos de $1200 \mathrm{mg}$ (IOM, 1997), portanto, apenas $3,30 \mathrm{~g}$ e $3,97 \mathrm{~g}$ de casca de ovo em pó, respectivamente, supririam essas necessidades diárias de cálcio, sem considerar a biodisponibilidade desse mineral na fonte em questão. 
Para suprir as demandas nutricionais de outros minerais, tais como fósforo, magnésio, potássio e sódio haveria a necessidade de ingestão de grandes quantidades de casca de ovo em pó (Tabela 4).

\section{TABELA 4 - NECESSIDADE DE CONSUMO DE CASCA DE OVO EM PÓ (g) PARA ATENDER AS DEMANDAS NUTRICIONAIS DE MAGNÉSIO, SÓDIO, FÓSFORO E POTÁSSIO PARA ADULTOS}

\begin{tabular}{c|c|c}
\hline Minerais & Recomendação diária $(\mathrm{mg})$ & $\begin{array}{c}\text { Consumo de casca de ovo } \\
\text { em pó }(\mathrm{g}) \text { para suprir as } \\
\text { recomendações diárias }\end{array}$ \\
\hline Fósforo & $700^{*}$ & 712,8 \\
Magnésio & Homens: $19-30$ anos: $400^{*}$ & 113,9 \\
& $\geq 31$ anos: $420^{*}$ & 119,6 \\
& Mulheres: $19-30$ anos: $310^{*}$ & 88,3 \\
Potássio & $\geq 31$ anos: $320^{\star}$ & 91,1 \\
Sódio & $4700^{* *}$ & $11.190,5$ \\
& $1500^{* *}$ & 1276,6 \\
\hline
\end{tabular}

"IOM, 1997; *"IOM, 2004.

Vale lembrar que o consumo de elevada quantidade do produto não seria interessante, pois a ingestão de cálcio acima dos níveis recomendados parece não contribuir, proporcionalmente, para a deposição de cálcio no tecido ósseo (SILVA, TEIXEIRA e GOLDBERG, 2004).

Kimura (2002) avaliou ratos Wistar que consumiram ração suplementada com doses de cálcio 3 a 4 vezes superiores às recomendadas e observou redução do conteúdo ósseo mineral, sobrecarga renal e enrijecimento arterial. Sabendo-se que a casca de ovo apresenta grande quantidade em cálcio, talvez deva ser consumida em quantidades suficientes para atender apenas as necessidades nutricionais.

É fundamental salientar a importância deste estudo, principalmente com relação aos altos níveis de cálcio detectados no produto, que possivelmente pode atuar como fonte alternativa de cálcio. Além do aspecto econômico e ecológico, o cálcio da casca de ovo apresenta vantagens nutricionais por não estar associado a elevadas quantidades de proteína e sódio. Isso ocorre com os queijos que podem induzir aumento da excreção renal de cálcio (IOM, 1997; WEINSIER e KRUMDIECK, 2000).

Segundo Lerner et al. (2000), Albuquerque e Monteiro (2002) e Silva, Teixeira e Goldberg (2004), crianças e adolescentes de diferentes regiões brasileiras não consomem quantidade suficiente de cálcio para atingir desenvolvimento desejável, faixa etária que exige maior aporte desse mineral para garantir boa formação óssea.

A ingestão deficiente de cálcio na infância e na adolescência predispõe à osteoporose, doença de alto impacto econômico que se manifesta nos idosos (MARQUES-NETO, 2001). Considerando a importância da ingestão adequada desse mineral, o consumo da casca de ovo em pó poderia contribuir positivamente para a complementação do cálcio em dietas deficientes desse mineral, prevenindo futuramente a osteoporose. Entretanto, seu consumo deve ser controlado para que não ultrapasse os níveis máximos de ingestão recomendados por faixa etária.

\section{CONCLUSÃO}

A casca de ovo de galinha em pó pode ser considerada como material rico em minerais, especialmente no que diz respeito ao cálcio, micronutriente bastante relevante para a saúde óssea. 
Além disso, trata-se de fonte economicamente viável, de fácil acesso e que praticamente não contribui energeticamente na dieta.

O produto obtido deve ser estudado como possível fonte de cálcio para pacientes portadores de fenilcetonúria, cujos teores de fenilalanina na alimentação têm que ser baixos, característica obtida no produto avaliado neste estudo.

\section{ABSTRACT}

\section{CHEMICAL COMPOSITION OF CHICKEN EGGSHELL POWDER}

Chicken eggshell is a by-product used as an ingredient of food multimixtures distributed in Brazil. The lack of chemical information about eggshell inspired this study aiming the development of technology to obtain this product in powder form and determine its nutrients content with the intend of using it like a calcium source. After sanitized and dried, the eggshells were turned into powder using a mill, and obtained a profit of $98.45 \pm 0.70 \%$. Analytical determinations showed that $100 \mathrm{~g}$ of this product contained low moisture $(1.3490$ $\pm 0.0274 \mathrm{~g})$, ash equivalent to $91.9600 \pm 0.2218 \mathrm{~g}$ and low caloric value $(30.6823 \pm 4.9069 \mathrm{Kcal})$, originated from $4.3693 \pm 0.3977 \mathrm{~g}$ of proteins, $0.7837 \pm 0.0560 \mathrm{~g}$ of lipids and $1.5379 \pm 0.7029 \mathrm{~g}$ of total carbohydrates, and also $30.263,72 \mathrm{mg}$ of calcium. Considering that calcium daily intake for healthy adults is $1000 \mathrm{mg}$ and, $3.30 \mathrm{~g}$ of eggshell powder would provide this dietary requirement. The results suggest that this by-product is a potential source of calcium, but further studies concerning its biodisponibility are still recommended.

KEY-WORDS: CALCIUM; EGGSHELL; Gallus gallus.

\section{REFERÊNCIAS}

1 ALBUQUERQUE, M.F.M.; MONTEIRO, A.M. Ingestão de alimentos e adequação de nutrientes no final da infância. Revista de Nutrição, v. 15, p.291-299, 2002

2 ANDRIGUETTO, J.M.; PERLY, L.; MINARDI, I.; GEMAEL, A.; FLEMMING, J.S.; SOUZA, G.A.; BONA FILHO, A. Nutrição animal, as bases e os fundamentos da nutrição animal: os alimentos. São Paulo: Editora Nobel, 1999395 p.

3 AOAC. Association of Official Analytical Chemists. Official methods of analysis of AOAC international. $17^{\text {th }}$ ed. Washington, 2000.

4 BRASIL. Ministério da Saúde. Portaria nº 847 de 31 de outubro de 2002. Aprova o protocolo clínico e diretrizes terapêuticas - fenilcetonúria - fórmulas de aminoácidos isenta de fenilalanina. Diário Oficial [da] República Federativa do Brasil, Brasília, Seção 1, p.83, 2002.

5 FISHBEIN, L. Multiple sources of dietary calcium - some aspects of its essentiality. Regulatory Toxicology and Pharmacology, New York, v. 39, p. 67-80, 2004.

6 FAO/WHO. Food and Agriculture Organization/World Health Organization. Expert Consultation. Energy and protein requirements. Geneva, 1985. p. 65 (Technical Reports Series, 724).

7 IOM. Institute of Medicine. Dietary reference intakes for calcium, phosphorus, magnesium, vitamin D and fluoride. Washington: National Academy Press, 1997. 448 p.

8 IOM. Institute of Medicine. Dietary reference intakes for water, potassium, sodium, chloride and sulfate. Washington: National Academy Press, 2004. 610 p.

9 IAL. Instituto Adolfo Lutz. Normas analíticas do Instituto Adolfo Lutz: métodos químicos e físicos para análise de alimentos. 4.ed. São Paulo, 2005.

10 IBGE. Instituto Brasileiro de Geografia e Estatística. Pesquisa da produção de ovos de galinha no Brasil em 2008. Disponível em:<http://www.ibge.gov.br>. Acesso em: 22 jan. 2009.

11 KIMURA, M. Osteoporosis induced by over calcium intake. American Journal Clin. Nutr., v. 75, p. 384-392, 2002.

12 KRAUSE, M.V.; MAHAN, L.K. Alimentos, nutrição \& dietoterapia. 6. ed. São Paulo: Ed. Roca, 2002. 981 p.

13 LERNER, B.R.; LEI, D.L.M.; CHAVES, S.P.; FREIRE, R.D. O cálcio consumido por adolescentes de escolas públicas de Osasco, São Paulo. Revista de Nutrição, v.13, p.57-63, 2000. 
14 MARQUES-NETO, J. F. Resultados da campanha nacional de prevenção da osteoporose. São Paulo: Merck Sharp \& Dohme, BG Cultural, 2001. p.15-52.

15 NAVES, M.M.V. Pó da casca de ovo como fonte de cálcio: qualidade nutricional e contribuição para o aporte adequado de cálcio. Revista da UFG, Goiânia, v.5, n.1, p.24-26, 2003.

16 ORNELLAS, L. H. Técnica dietética: seleção e preparo de alimentos. 7.ed. São Paulo: Ed. Atheneu, 2001.330 p.

17 SANT'ANA, L.F.R; COSTA, N.M.B.; OLIVEIRA, M.G.A.; GOMES, M.R.A. Valor nutritivo e fatores antinutricionais de multimisturas utilizadas como alternativa alimentar. Brazilian Journal of Food Technology, v.3, p.129-135, 2000.

18 SILVA, C.C.; TEIXEIRA, A.S.; GOLDBERG, T.B.L. Impacto da ingestão de cálcio sobre a mineralização óssea em adolescentes. Revista de Nutrição, v. 17, n. 3, p. 351-359, 2004.

19 SPACKMAN, D.H.; STEIN, W.H.; MOORE, S. Automatic recording apparatus for use in the chromatography of amino acids. Analytical Chemistry, v.30, p.1190-1206, 1958.

20 TRIOLA, M. F. Introdução à estatística. 9.ed. Rio de Janeiro: Livros Técnicos e Científicos, 2005.682 p.

21 UBA. União Brasileira de Avicultura. Consumo de ovos: em 2006, o brasileiro consumiu 141 ovos. Disponível em: <http://www.aviculturaindustrial.com.br>. Acesso em: 15 nov. 2008.

22 WEAVER, C.M.; HEANEY, R.P. Cálcio. In: SHILS, M.E.; OLSON, J.A.; SHIKE, M., ROSS, A.C. (eds). Tratado de nutrição moderna na saúde e na doença. 9. ed. São Paulo: Manole, 2003. p.153-67.

23 WEINSIER, R.L.; KRUMDIECK, C.L. Dairy foods and bone health: examination of the evidence. Am. J. Clin. Nutr., v. 72, n. 3, p. 681-689, 2000.

\section{Agradecimentos}

Ao CNPq pela concessão da bolsa de estudos. 